\title{
Increased DEF6 expression is correlated with metastasis and poor prognosis in human osteosarcoma
}

\author{
QIAO ZHANG ${ }^{1 *}$, GUO-SHENG ZHAO $^{2 *}$, YA CAO $^{3}$, XUE-FENG TANG $^{3}$, \\ QIU-LIN TAN ${ }^{3}$, LU LIN $^{2}$ and QIAO-NAN GUO ${ }^{3}$ \\ ${ }^{1}$ Department of Pain and Rehabilitation, Xinqiao Hospital, Army Medical University, Chongqing 400037; \\ ${ }^{2}$ Department of Orthopedic Surgery, The Second Affiliated Hospital of Chongqing Medical University, Chongqing 400010; \\ ${ }^{3}$ Department of Pathology, Xinqiao Hospital, Army Military Medical University, Chongqing 400037, P.R. China
}

Received November 27, 2019; Accepted May 14, 2020

DOI: $10.3892 / 01.2020 .11743$

\begin{abstract}
Metastasis is the primary cause of high mortality in patients with osteosarcoma (OS). However, the molecular mechanisms underlying the regulation of metastatic disease are yet to be determined. Differentially expressed in FDCP 6 homolog (DEF6) has been demonstrated to be correlated with the metastatic behavior of several cancers, such as breast, ovarian and colorectal cancers. However, the role of DEF6 in OS remains unknown. Accordingly, the current study aimed to investigate the relationship between DEF6 expression and the malignant behavior of OS. The results revealed that high levels of DEF6 in OS tissues were associated with advanced clinical stage and metastases. Furthermore, immunohistochemistry results predicted a poor prognosis in 58 human OS specimens. Additionally, DEF6 expression was reported to be upregulated in human OS cell lines compared with a normal osteoblast cell line. small interfering RNA transfection, cell proliferation and colony formation assays, wound healing assays and Transwell assays were performed. DEF6 was not identified to be a major driver of OS cell proliferation, but it significantly contributed to metastatic potential in vitro. In addition, bioinformatics, western blotting and immunohistochemistry results indicated that MMP9 expression was positively correlated with DEF6 expression in human OS. To summarize, the results revealed that increased levels of DEF6 were associated with metastasis and poor prognosis in human OS and that DEF6 expression is positively correlated with MMP9 expression. The results indicated that DEF6 may serve as a potential antimetastatic target for OS.
\end{abstract}

Correspondence to: Professor Qiao-Nan Guo, Department of Pathology, Xinqiao Hospital, Army Military Medical University, 183 Xinqiaozhengjie Raod, Chongqing 400037, P.R. China E-mail: qiaonan85@263.net

*Contributed equally

Key words: differentially expressed in FDCP 6 homolog, osteosarcoma, metastasis, matrix metallopeptidase P9

\section{Introduction}

Osteosarcoma (OS) is generally a high-grade tumor that exhibits locally aggressive behavior and causes early systemic metastases (1). OS primarily occurs in the long bones of young adults and is the most common primary bone sarcoma worldwide (1). The 5 year relative survival rate of early stage OS in the US was 20-30\% in children and adolescents diagnosed in the early 1970s [1970-1973; (2)]. This increased to $64 \%$ in those diagnosed during 1986-1993, a figure which may be attributed to the combined use of multiagent or neoadjuvant chemotherapy and advanced surgery (3). However, in the past $>20$ years, no significant improvements have been recorded in the 5 year survival rate of OS: The 5-year relative survival rate of OS was $\sim 65-70 \%$ in children and adolescents diagnosed between 2008-2014 (4). Metastasis is primarily responsible for an impediment in treatment development and can be held accountable for therapeutic failure in patients with OS (5). There is a lack of effective strategies to treat advanced OS and the 5-year survival rate of patients with distant metastases was $\sim 20-30 \%$ in the US and Italy during 1980-2000 (6,7). Thus, recent studies have focused on identifying molecular markers that could be used for early metastasis detection and prognosis assessment, serving as potential therapeutic targets for advanced OS (3,5,8-10).

Differentially expressed in FDCP 6 homolog (DEF6) and associated homologous genes (Def2, Def3, Def8) were first described as novel mouse genes expressed in the hemopoietic system (11). DEF6, also known as IFN regulatory factor 4-binding protein (IBP), encodes an interferon regulatory factor 4 binding protein and is diffusely expressed in the human immune system (12). DEF6 is predominantly expressed in $\mathrm{T}$ lymphocytes and regulates several $\mathrm{T}$-cell processes, including T-helper-1/2/17 differentiation and the coordination of actin cytoskeleton remodeling (13-15). DEF6 serves a critical and unique role in regulating systemic autoimmunity as a SWEF family, which is a small and unique protein family of Rho guanine nucleotide exchange factors (GEF), only comprised of DEF6 and SWAP-70, controlling both cytoskeletal dynamics and the activity of IFN regulatory factor 4 (IRF4) (13). Additionally, DEF6 reportedly regulates bone remodeling by restraining osteoclastogenesis 
and inflammatory bone resorption (16). Although numerous studies have focused on the role of DEF6 in the immune system, abnormal DEF6 expression levels in non-immune cells, particularly tumor cells, indicate a potential function of DEF6 in tumorigenesis regulation $(12,17,18)$. DEF6 has been reported to be highly expressed in breast carcinoma, ovarian cancer, colorectal cancer, oral squamous cell carcinoma and extraskeletal myxoid chondrosarcoma (17-21), exhibiting various malignant behaviors and cancer cell biology, including proliferation (19), autophagy (22), invasion (20) and epithelial-mesenchymal transition (12). Additionally, Rho-GTPase and mammalian target of rapamycin (mTOR) signaling pathways, which serve a key role in OS tumorigenesis and metastasis $(3,8)$, are targets of DEF6 that facilitate tumor cell metastasis and enhance tumorigenic potential $(12,22)$. However, the expression and biological functions of DEF6 in OS are yet to be elucidated.

In the present study, the role of DEF6 with regards to clinicopathological features of patients and its prognostic value in human OS was investigated. DEF6 expression levels were determined in OS and normal osteoblast cell lines. Furthermore, DEF6 was demonstrated to act as an oncogenic driver that promoted the metastatic potential of OS cells. To the best of our knowledge, this is the first study to demonstrate the role of DEF6 in OS.

\section{Materials and methods}

Human specimens. Specimens of human osteosarcoma tissues were collected from 58 patients with pathologically confirmed OS at the Southwest Hospital and Xinqiao Hospital of the Army Medical University from February 2011 to November 2015. The inclusion criteria were as follows: No patients received pre-operative anticancer treatment before pathological confirmation of OS and all patients received standard treatment (chemotherapy and surgery) following pathologically confirmation of OS. The exclusion criteria were as follows: Patients who had other fatal diseases or cancer and non-cancer associated mortality. Given that the paired normal OS tissues were not completely collected during surgery, human benign bone tumor specimens were used as the control group and compared with the OS specimens in the present study. The specimens were obtained from 12 patients with histopathologically-confirmed osteoblastoma from Xinqiao Hospital from March 2015 to November 2017. The clinical staging and corresponding treatment for OS were based on the Enneking system (23). Patients were classified as with or without developed distant metastasis at diagnosis or following treatment. The clinicopathological features of all patients with osteosarcoma are listed in Table I. Among the 58 patients with OS, 24 were women and 34 were men. The median age of these patients was 22 years (age range, 8-59 years). A total of 22 patients with primary osteosarcoma had recurrence following surgery and received chemotherapy, 12 patients had metastasis at first diagnosis, and eight more patients developed distant metastasis following treatment. Written informed consent was obtained from all patients or their guardians. All experiments were approved by the Ethics Committees of Southwest Hospital (approval no. 27-2011) and Xinqiao Hospital (approval no. 2018-069-01).
Cell culture. MNNG/HOS, SaOS2, MG63 and U2OS OS cell lines were purchased from Cellcook Biological Technology Co., Ltd (www.cellcook.com). The human osteoblast cell line hFOB1.19 was obtained from the Cell Type Culture Collection of the Chinese Academy of Sciences. A malignant transformed hFOB1.19 cell line (MTF cells) was produced in the laboratory (24). MNNG/HOS, SaOS2, MG63, U2OS and MTF cells were cultured in high-glucose DMEM (Hyclone; Cytiva) supplemented with 10\% FBS (Lonsera Science SRL) and $1 \%$ penicillin-streptomycin (Hyclone; Cytiva), and maintained at $37^{\circ} \mathrm{C}$ and $5 \% \mathrm{CO}_{2}$. hFOB1.19 cells were cultured in DMEM/F12 (Hyclone; Cytiva) with 15\% FBS (Lonsera Science SRL) and maintained at $35^{\circ} \mathrm{C}$ and $5 \% \mathrm{CO}_{2}$. Both cells were cultured to the logarithmic growth phase of the third generation prior to use for subsequent experimentation, and the medium was changed every 48-72 $\mathrm{h}$.

Antibodies. Primary mouse anti-human DEF6 antibody (cat. no.FAB-KY015-IBP) were provided by Professor Chuanmin $\mathrm{Hu}$ (Army Medical University), which were produced and verified in their laboratory (21). Primary rabbit anti-human matrix metallopeptidase (MMP9) antibody (cat. no. 13667) was purchased from Cell Signaling Technology, Inc. (CST) and primary rabbit anti-human GAPDH antibody (cat. no. AB-P-R001) was purchased from Hangzhou Goodhere Biotech Co., Ltd.

Immunohistochemistry (IHC). IHC was performed using a kit (Beijing Zhongshan Golden Bridge Biotechnology Co. Ltd., ZSGB-BIO, Beijing, China), as previously described (8). Briefly, human OS or osteoblastoma tissue sections were deparaffinized in two replicate bottles of xylene for 10 min each at room temperature. Tissue section were subsequently rehydrated with a graded series of ethanol and then antigens were retrieved in $10 \mathrm{mM}$ citrate buffer by boiling for $10 \mathrm{~min}$, and cooling to room temperature. Sections were blocked with a ready to use goat serum solution in the UltraSensitive ${ }^{\mathrm{TM}} \mathrm{SP}$ (mouse/rabbit) IHC kit (cat. no. KIT-9720; Fuzhou Maixin Biotech Co., Ltd.) for $\sim 30 \mathrm{~min}$ at $37^{\circ} \mathrm{C}$ and incubated with $3 \%$ hydrogen peroxide for $\sim 15 \mathrm{~min}$ at $37^{\circ} \mathrm{C}$ to inhibit endogenous peroxidase activity. Tissue sections were subsequently incubated with primary antibodies against DEF6 (cat. no. FAB-KY015-IBP) and MMP9 (cat. no. 13667, CST), dilution by primary antibody dilution buffer (cat. no. ZLI-9028; ZSGB-BIO) overnight at $4^{\circ} \mathrm{C}$. Subsequently, sections were incubated with ready-to-use undiluted secondary antibodies conjugated with biotin in the UltraSensitiveTM SP (mouse/rabbit) IHC kit (cat. no. KIT-9720; Fuzhou Maixin Biotech Co., Ltd.) for $30 \mathrm{~min}$ at $37^{\circ} \mathrm{C}$. Subsequently, DAB staining (DAB kit; cat. no. DAB-0031; Fuzhou Maixin Biotech Co., Ltd.) was used for $5 \mathrm{~min}$ at room temperature and the nuclei were stained with hematoxylin for $2.5 \mathrm{~min}$ at room temperature. The stained sections were observed under a light microscope (Olympus Corporation, magnification, $\mathrm{x} 400$ ). For quantitative assessment of DEF6 (1:500; cat. no. FAB-KY015-IBP) protein expression in primary site tissues or metastatic site tissues of OS, the percentage of positive cells was determined in 5 randomly selected fields of view using a light microscope and higher-magnification objectives (magnification, $x 400$ ), including $\geq 50$ cells. The final IHC score was a product of the positive cell ratio score $(0$, no immunoreactivity; $1, \leq 25 \%$ cells 
Table I. Correlation between DEF6 expression and clinicopathological features in patients with osteosarcoma.

\begin{tabular}{|c|c|c|c|}
\hline \multirow[b]{2}{*}{ Characteristic } & \multicolumn{2}{|c|}{ DEF6 expression } & \multirow[b]{2}{*}{$\chi^{2}$} \\
\hline & High & Low & \\
\hline Age, years & & & 0.808 \\
\hline$\leq 30$ & 26 & 19 & \\
\hline$>30$ & 8 & 5 & \\
\hline Sex & & & 0.097 \\
\hline Male & 23 & 11 & \\
\hline Female & 11 & 13 & \\
\hline Clinical stage & & & $0.033^{\mathrm{a}}$ \\
\hline IIA & 7 & 7 & \\
\hline IIB & 16 & 16 & \\
\hline III & 11 & 1 & \\
\hline Local recurrence & & & 0.544 \\
\hline Yes & 14 & 8 & \\
\hline No & 20 & 16 & \\
\hline Distant metastasis & & & $0.003^{\mathrm{a}}$ \\
\hline Yes & 17 & 3 & \\
\hline No & 17 & 21 & \\
\hline Histological type & & & 0.462 \\
\hline Osteoblastic & 17 & 17 & \\
\hline Chondroblastic & 13 & 5 & \\
\hline Fibroblastic & 2 & 1 & \\
\hline Others & 2 & 1 & \\
\hline Tumor size & & & 0.233 \\
\hline$<8 \mathrm{~cm}$ & 22 & 19 & \\
\hline$\geq 8 \mathrm{~cm}$ & 12 & 5 & \\
\hline Tumor location & & & 0.085 \\
\hline Limbs & 32 & 19 & \\
\hline Others & 2 & 5 & \\
\hline
\end{tabular}

aP $<0.05$. DEF6, differentially expressed in FDCP 6 homolog.

stained; $2,26-50 \%$ cells stained; $3,51-75 \%$ cells stained; 4 , $\geq 76 \%$ cells stained) and relative expression score $(0$, negative; 1 , light yellow staining; 2 , yellow staining; 3 , brown or dark brown staining). Final scores of $\leq 1$ indicated negative expression of DEF6, scores between $>1$ and $\leq 3$ indicated weak positive expression, scores between $>3$ and $<8$ indicated positive expression and scores $\geq 8$ indicated overexpression. Negative and weak positive expression of DEF6 represented low protein levels, while positive and overexpression of DEF6 represented high protein levels.

The semiquantitative assessment of MMP9 (1:200; cat. no. 13667; Cell Signaling Technology, Inc.) protein expression was then performed. Positive MMP9 staining was observed under at least 5 fields of view, with $>10$ OS cells around tumor angiosomes, using a light microscope (magnification, $\mathrm{x} 400$ ).

Transfection of short interfering RNAs (siRNAs). Small interfering (si)RNAs targeting DEF6 were purchased from
Guangzhou RiboBio Co., Ltd. For transient knockdown experiments, MTF and MNNG/HOS cells (exhibit significantly higher DEF6 expression compared with other OS cells) were transfected with $30 \mathrm{nM}$ targeting or scrambled DEF6 siRNAs using the RiboBio-FECT ${ }^{\mathrm{TM}} \mathrm{CP}$ kit (Guangzhou RiboBio Co., Ltd.), according to manufacturer's protocol. Knockdown efficiency was assessed 48 or $72 \mathrm{~h}$ post-transfection via RT-qPCR and western blotting analyses. The siRNA target sequences used were DEF6-siRNA-1 (5'-ACAGTATGCTCT CCAATCA-3') and DEF6-siRNA-2 (5'-CTGCTACTTTGG GAGTGAA-3').

Reverse transcription quantitative PCR (RT-qPCR). Following appropriate treatments [transfection with short interfering (si) RNAs], total RNA was extracted from osteosarcoma cells using RNAiso $^{\text {TM }}$ Plus (Takara Biotechnology Co., Ltd.). RNA samples were then reverse transcribed into cDNA using a QuantScript RT kit (Takara), according to manufacturer's protocol. RT-qPCR was performed using a SYBR Premix kit (Takara), 
according to the manufacturer's protocol. The thermocycling conditions were used as previously described (25), as follows: $95^{\circ} \mathrm{C}$ for $30 \mathrm{sec}$, followed by 40 cycles at $95^{\circ} \mathrm{C}$ for $15 \mathrm{sec}$ and $60^{\circ} \mathrm{C}$ for $60 \mathrm{sec}$. The following primer sequences were used for qPCR: DEF6 forward, 5'-GAAAGCTCGGCGAGATGAAG-3' and reverse, 5'-GATGTAGCGCTCCTGCTCCT-3'; MMP9 forward, 5'-AAACCGAGTTGGAACCACGAC-3' and reverse, 5'-AGACGGGTATCCCTTCGACG-3'; and GAPDH forward, 5'-CTTTGGTATCGTGGAAGGACTC-3' and reverse, 5'-GTA GAGGCAGGGATGATGTTCT-3'. Relative expression levels were calculated using the $2^{-\Delta \Delta C q}$ method (26).

Cell proliferation and colony formation assays. Cell proliferation assays were performed using a Cell Counting Kit- 8 (CCK-8; cat. no. C0038; Beyotime Institute of Biotechnology), as previously reported (27). Cells (MNNG/HOS and MTF) were digested using Trypsin (Hyclone; Cytiva) and seeded into four 96 -well plates $\left(1.5 \times 10^{3}\right.$ cells/well) following siRNA transfection. Following incubation for 24, 48, 72 and $96 \mathrm{~h}$, at $37^{\circ} \mathrm{C}$ in $5 \% \mathrm{CO}_{2}, \mathrm{CCK}-8$ reagent mixed with serum-free DMEM (1:9) $100 \mu \mathrm{l}$ was added and five wells with no cell were selected as blank control also added $100 \mu \mathrm{l}$ CCK- 8 reagent mixed with serum-free DMEM. Both of them were followed by incubation for $1.5 \mathrm{~h}$ at $37^{\circ} \mathrm{C}$ in $5 \% \mathrm{CO}_{2}$. A plate reader (Thermo Fisher Scientific, Inc.) was used to measure the optical density (OD) at $450 \mathrm{~nm}$. Final OD values were obtained by subtracting the OD values of cell-containing wells from blank wells, using he following formula: $\mathrm{OD}_{\text {Final }}=\mathrm{OD}_{\text {measured }}-\mathrm{OD}_{\text {blank }}\left(\mathrm{OD}_{\text {blank }}\right.$ refers to the OD values of wells maintained in $100 \mu \mathrm{l}$ CCK-8 reagent and serum-free DMEM).

Colony formation assays were performed as previously described (28). MTF and MNNG/HOS cells, which were transfected with either scrambled siRNAs or DEF6 siRNA, were plated in six-well plates (100 cells/well) and incubated at $37^{\circ} \mathrm{C}$. On day 11 for MTF and on day 13 for MNNG/HOS cells, cells were fixed with $4 \%$ paraformaldehyde for $15 \mathrm{~min}$ at room temperature and then stained with $0.1 \%$ crystal violet (Beyotime Institute of Biotechnology) for $5 \mathrm{~min}$ at room temperature. After rinsing with water, colony numbers ( $>50$ cells) were determined.

Wound healing assay. The wound healing assay was performed as previously described (8). Transfected MNNG/HOS and MTF cells $\left(\sim 6 \times 10^{5}\right.$ cells/well) were seeded in six-well plates and cultured until they reached $80 \%$ confluence. A $100 \mu 1$ micropipette tip was used to create a wound. Cells were monitored at 0 and $24 \mathrm{~h}(\mathrm{MNNG} / \mathrm{HOS})$ or $48 \mathrm{~h}$ (MTF) following wounding and images of wound healing were captured using an inverted phase-contrast light microscope (Olympus Corporation; magnification, x100) equipped with DP Controller software (version 3.1.1.267; Olympus Life Science; Olympus Corporation).

Transwell invasion assay. Transfected cells were resuspended in serum-free DMEM, after which $200 \mu 1$ of the cell suspension was seeded $\left(2 \times 10^{5}\right.$ cells $\left./ \mathrm{ml}\right)$ into the upper chambers of an $8 \mu \mathrm{m}$ Transwell filter (Merck KGaA), that were precoated with 1:3 diluted Matrigel (BD Biosciences). DMEM supplemented with $10 \%$ FBS $(600 \mu 1)$ was added to the lower chamber and incubated for $24 \mathrm{~h}$ at $37^{\circ} \mathrm{C}$ in $5 \% \mathrm{CO}_{2}$. Subsequently, invaded cells were fixed with $4 \%$ paraformaldehyde for $15 \mathrm{~min}$ at room temperature and stained with $0.1 \%$ crystal violet for $5 \mathrm{~min}$ at room temperature. Cell counting was performed in at least 5 randomly selected fields of view under an inverted phase-contrast light microscope (Olympus Corporation; magnification, $\mathrm{x} 200$ ).

Western blotting. As previously reported (29), cells (MG63, hFOB1.19, SaOS2, U2OS, MNNG/HOS and MTF) were collected following transfection and treated with M-PER ${ }^{\mathrm{TM}}$ Mammalian Protein Extraction Reagent (cat. no. 78501; Thermo Fisher Scientific, Inc.) containing a protease inhibitor (Roche Diagnostics, Co. Ltd.). Protein concentration was determined using the BCA Protein Assay kit (cat. no. P0010S; Beyotime Institute of Biotechnology). Proteins (40-50 $\mu \mathrm{g} /$ lane) were subsequently separated using $10 \%$ SDS Tris-glycine gels and transferred onto PVDF membranes [cat. no. 3010040001; Roche Diagnostics (Shanghai) Co., Ltd.]. The membranes were blocked with 5\% fat-free milk and incubated at $4{ }^{\circ} \mathrm{C}$ with the following primary antibodies overnight: GAPDH $(1: 1,000)$, DEF6 $(1: 1,000)$ and MMP9 (1:400) were used. All primary antibodies were diluted to the aforementioned concentration using Western Primary Antibody Dilution buffer (cat. no. P0023A; Beyotime Institute of Biotechnology). After rinsing with PBST. Secondary antibodies (goat anti-rabbit or goat anti-mouse IgG conjugated with HRP; 1:5,000; cat. nos. bs-0295G-HRP and bs-0368G-HRP; BIOSS) were subsequently applied and incubated for $1 \mathrm{~h}$ at room temperature. Immunoreactivity was detected using an ECL kit (BeyoECL Moon; Beyotime Institute of Biotechnology).

Analysis using the $R 2$ database. The $\mathrm{R} 2$ database (hgserver1.amc.nl) and subset data named 'mixed osteosarcoma-Kuijjer-127-vst-ilmnhwg6v2' were used for gene ontology (GO) analyses to investigate the relationship between DEF6 and other cancer related genes in patients with OS. The database was further used to analyze the correlation between DEF6, common MMP genes and tissue inhibitor of metalloproteinase (TIMP) genes associated with OS cell invasion (5).

Statistical analysis. Categorical data were analyzed using the $\chi^{2}$ test and Spearman's or Pearson's rank correlation coefficient. Quantitative data are presented as mean \pm standard deviation and analyzed using unpaired two-tailed Student's t-test or Mann-Whitney test (nonparametric test when P-value and $\mathrm{F}$ test $<0.05$ ) for between-group comparisons. ANOVA with Bonferroni's correction was used for comparing data in $>2$ groups. Spearman survival analysis was performed using the Kaplan-Meier method and log-rank test. $\mathrm{P}<0.05$ was considered to indicate a statistically significant difference. All analyses were performed using SPSS (version 20.0; IBM Corp.) or GraphPad Prism software (version 7.00; GraphPad Software, Inc.). All in vitro experiments were performed at least in triplicate.

\section{Results}

DEF6 is widely expressed in patients with OS. Previous studies have reported an abnormal expression of DEF6 in 
A
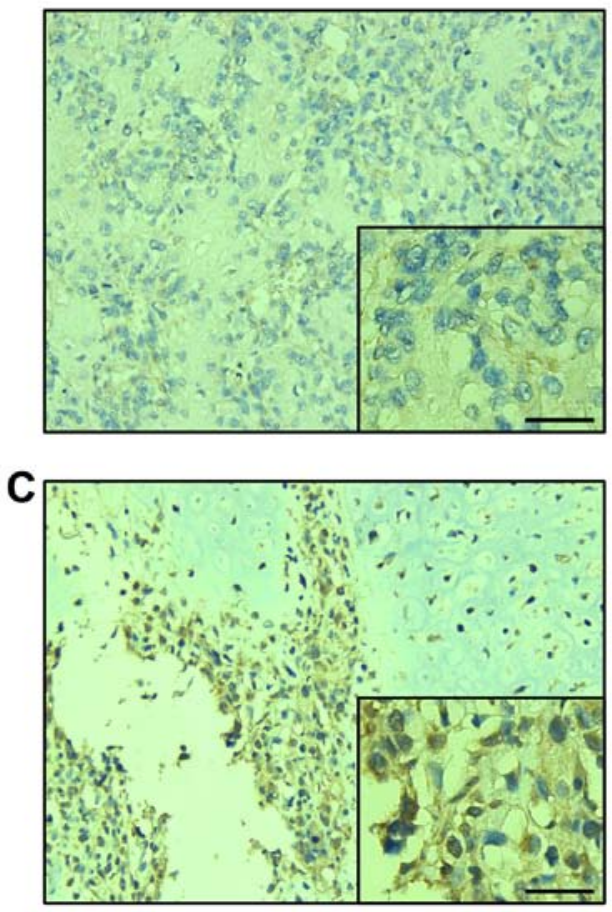

B

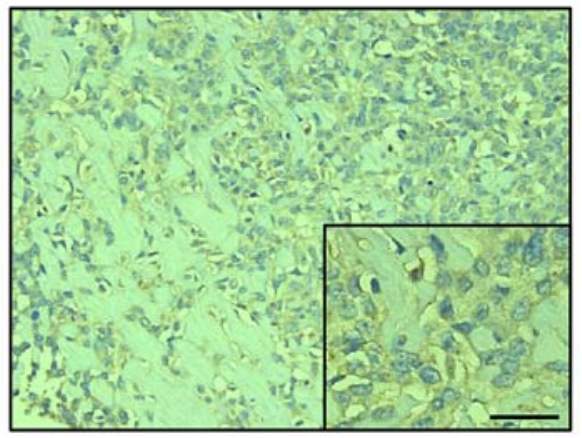

D

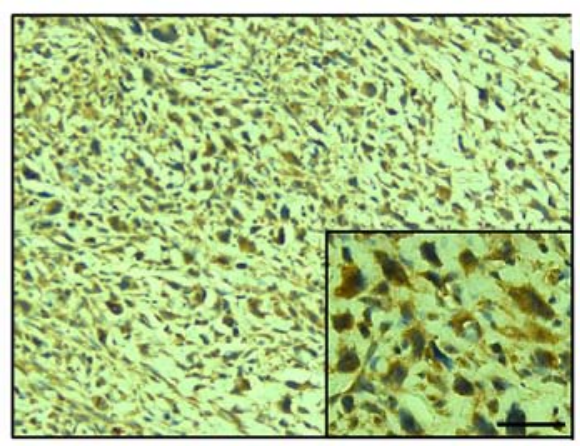

Figure 1. IHC was performed to determine DEF6 expression in human osteosarcoma tissues. (A) Negative expression of DEF6 (IHC score, $\leq 1$ ). (B) Weak positive expression of DEF6 (IHC score, $>1$ and $\leq 3$ ). (C) Positive expression of DEF6 (IHC score, $>3$ and $<8$ ). (D) Overexpression of DEF6 (IHC score, $\geq 8$ ). Magnifications, x200 and x400; scale bar, $25 \mu \mathrm{m}$. IHC, immunohistochemistry; DEF6, differentially expressed in FDCP 6 homolog.

multiple malignant tumors, indicating that DEF6 functions as an oncogene (17-21). IHC staining was performed to examine the expression of DEF6 in 58 human OS tissues and to determine the role of DEF6 in OS development. The results demonstrated that DEF6 was widely expressed in these tissues. A total of $0.05 \%$ cases exhibited negative staining for DEF6 (3/58; Fig. 1A), while $94.83 \%$ (55/58) cases exhibited positive staining for DEF6. Furthermore, $36.20 \%$ cases exhibited weak positive expression (21/58; Fig. 1B), 39.66\% exhibited positive expression (23/58; Fig. 1C) and $18.97 \%$ exhibited overexpression (11/58; Fig. 1D). As evidenced, DEF6 was expressed at high levels (positive expression/overexpression) in the majority of cases (34/58).

DEF6 is highly expressed, is positively associated with distant metastasis and predicts poor prognosis in patients with OS. IHC staining was utilized to examine DEF6 expression in 12 human osteoblastomas (benign bone tumors) and 58 OS tissues. As expected, DEF6 was observed to exhibit significantly higher expression $(\mathrm{P}<0.05)$ in $\mathrm{OS}$ compared with osteoblastoma tissues (Fig. 2A). Furthermore, the correlation between DEF6 expression and clinicopathological features of patients with OS via immunohistochemical staining was analyzed. The results demonstrated high DEF6 expression in $44.74 \%(17 / 38)$ of patients with OS and without metastasis, and $85.00 \%(17 / 20)$ in patients with OS and metastasis. This difference was statistically significant $(\mathrm{P}=0.003$; Table I). Accordingly, a high IHC score for DEF6 was observed more frequently in patients with distant metastatic disease (Fig. 2B). DEF6 was highly expressed at lung metastatic sites in samples from the case presented in Fig. 2B (Fig. 2C). Furthermore, a high DEF6 expression was significantly associated with worse clinical stage $(\mathrm{P}<0.05)$, but not with age, sex, location, size, local recurrence or histological subtype. Additionally, Kaplan-Meier curves indicated that the overall survival of patients with OS and high DEF6 expression levels was significantly shorter compared with OS and low DEF6 levels $(\mathrm{P}=0.021$; Fig. 2D). The results demonstrated that DEF6 was widely expressed in OS tissues and that high DEF6 levels were positively associated with metastasis and poor prognosis.

DEF6 is overexpressed in OS cell lines and contributes to metastatic potential in vitro. Western blotting was utilized to determine the expression of DEF6 in a series of cell lines. Excluding MG63 cells, DEF6 protein levels were significantly higher in OS cell lines compared with the osteoblast cell line hFOB1.19 (Fig. 3A). This validated that DEF6 that is widely expressed in OS tissues, with upregulated levels in OS cell lines. Furthermore, a significantly higher expression of DEF6 was exhibited in MTF cells compared with hFOB1.19 osteoblasts $(\mathrm{P}<0.001$, Fig. 3A), indicating that DEF6 upregulation is involved in the malignant transformation of osteoblasts. To determine whether increased levels of DEF6 contributed to the malignant proliferation of OS cells, DEF6 expression was knocked down using two siRNA sequences of DEF6 in MTF and MNNG/HOS cell lines (as they exhibit significantly higher DEF6 expression compared with other OS cells). Both target sequences demonstrated lower DEF6 protein levels, as demonstrated via western blotting (Fig. 3B). Furthermore, CCK-8 and colony formation assays were performed to investigate the proliferation of cells with or without DEF6 knockdown. The results demonstrated that DEF6 knockdown had no significant 
A

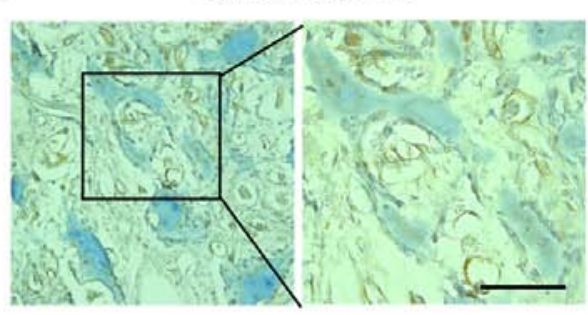

B

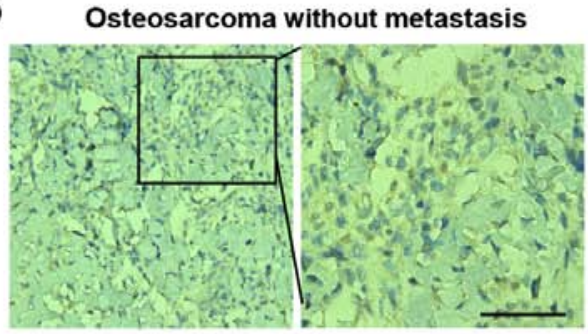

C

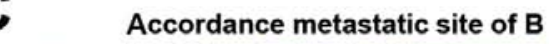

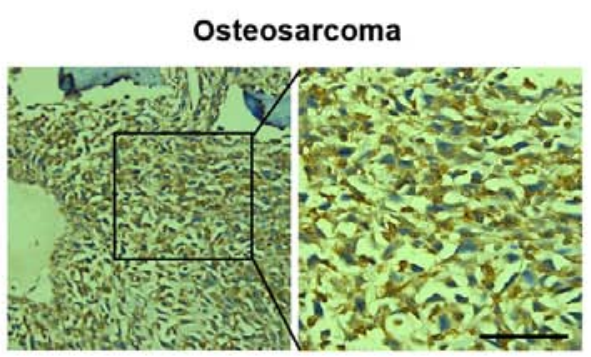
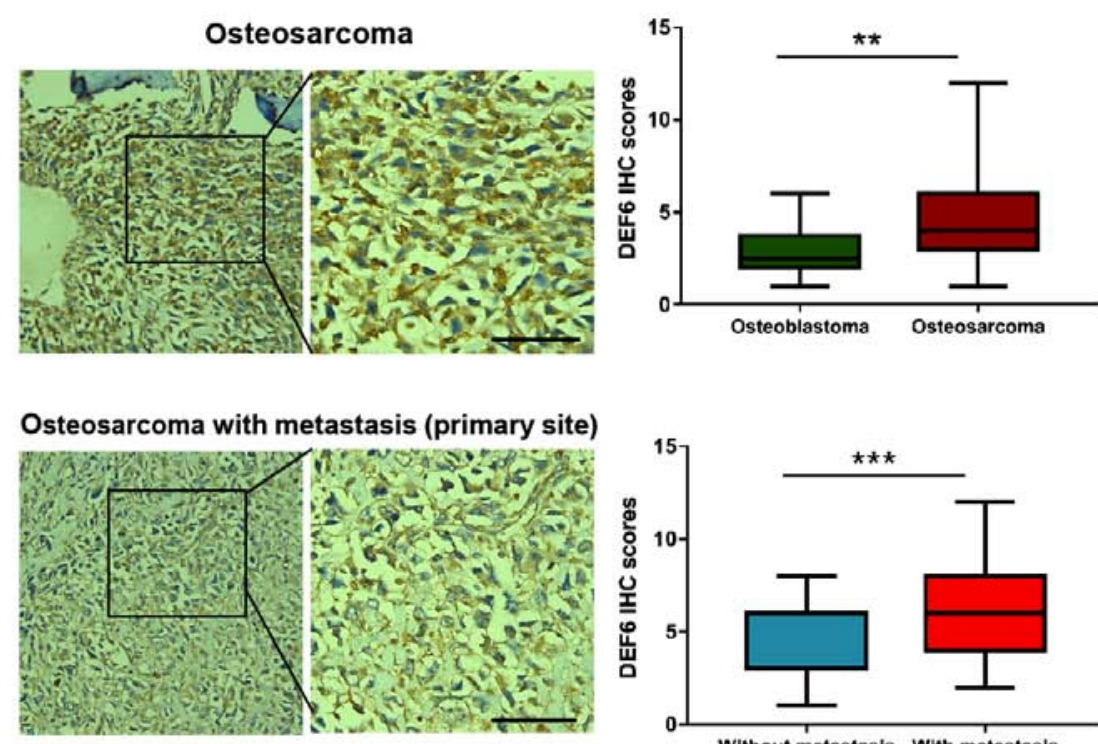

D

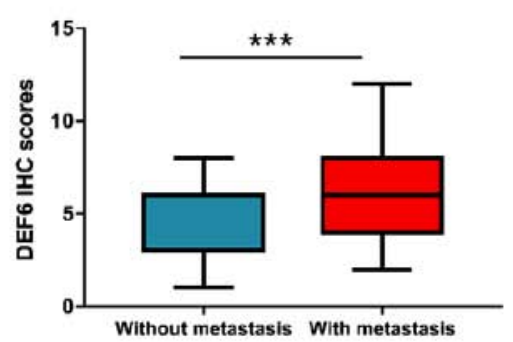

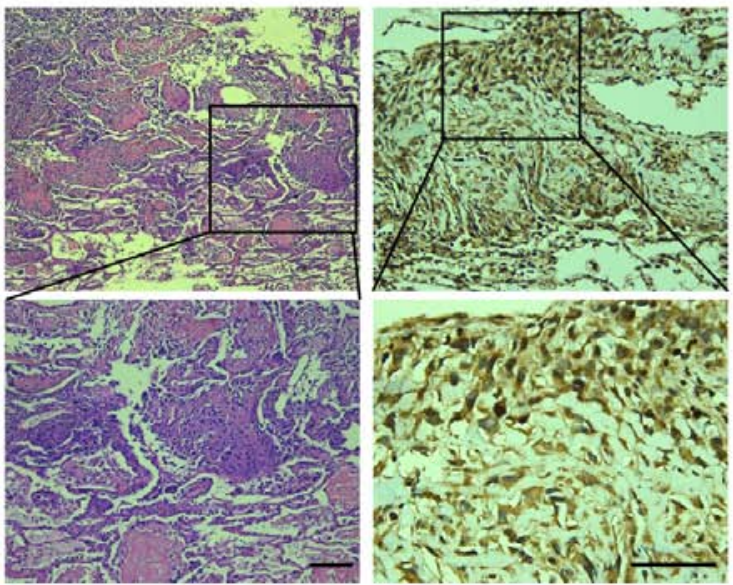

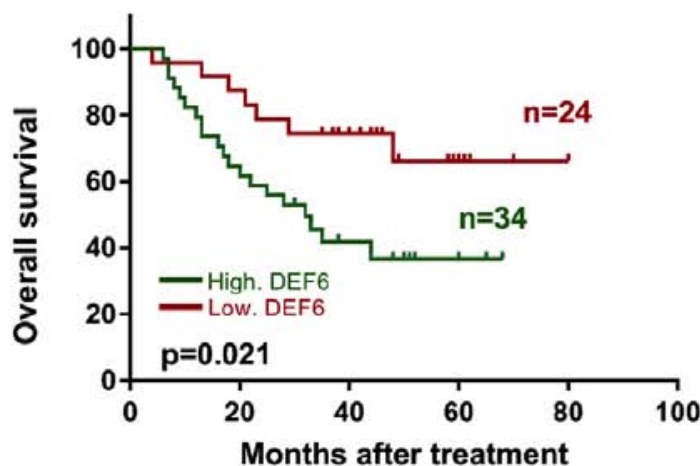

Figure 2. DEF6 is highly expressed in OS, positively associated with distant metastasis and predicts poor prognosis. (A) Representative images of IHC staining and quantitative comparison of IHC scores for DEF6 expression in osteoblastoma and OS. DEF6 expression was significantly higher in OS than in osteoblastoma. (B) Higher IHC scores of DEF6 were reported in patients with OS and metastasis compared with patients with OS without metastasis. (C) IHC staining for DEF6 expression of the OS lung metastatic site, as the primary site of OS was shown in Fig 2 B. Magnifications, x200 and x400. (D) Kaplan-Meier curve demonstrating that high levels of DEF6 predicted poor prognosis and shorter overall survival in patients with OS. ${ }^{* *} \mathrm{P}<0.01$ and ${ }^{* * *} \mathrm{P}<0.001$ as indicated. Scale bar, $50 \mu \mathrm{m}$. DEF6, differentially expressed in FDCP 6 homolog; OS, osteosarcoma; IHC, immunohistochemistry.

effect on the malignant proliferation of MTF or MNNG/HOS cells (Fig. 3C and D). Considering the positive association between DEF6 expression and metastasis in human OS tissues, wound healing and Transwell assays were performed to determine whether DEF6 knockdown suppressed cell motility. The results indicated that decreased levels of DEF6 in MTF and MNNG/HOS cells caused a significant reduction in cell migration and invasion, as evident from the decreased wound healing rates (Fig. 4A) and numbers of invading cells (Fig. 4B). In summary, the results indicated that although DEF6 may not be a major driver of OS cell proliferation, it significantly contributed to metastatic potential in vitro.

Knockdown of DEF6 expression significantly decreases MMP9 expression in OS cells in vitro. To further investigate how DEF6 influenced OS cell motility, the R2 database was used to identify a potential target of DEF6 in OS. As predicted, the results of GO analysis supported the aforementioned results of the current study and demonstrated DEF6 is more likely to facilitate OS cell migration and invasion rather than proliferation and survival (Fig. 5A). The results demonstrated that DEF6 was closely associated OS cell adhesion and motility. TIMPs reportedly serve a key role in regulating extracellular matrix remodeling, thereby affecting cell adhesion and motility (30). Additionally, MMPs and TIMPs reportedly have considerable influence on the invasive ability of cancer cells (31). Thus, the R2 database was used to investigate the correlation between DEF6 and MMPs or their inhibitor-associated genes, both of which have been widely reported to be correlated with OS metastases (10,30-36). The results revealed that there may be a positive correlation between DEF6 and MMP9 ( $\mathrm{P}<0.0001 ; \mathrm{r}=0.4278$; Fig. 5B). Furthermore, the results demonstrated that the mRNA and protein expression of MMP9 were significantly downregulated in association with a decrease in DEF6 expression in OS cell lines (Fig. 5C-D). Collectively, the results indicated that the 
A

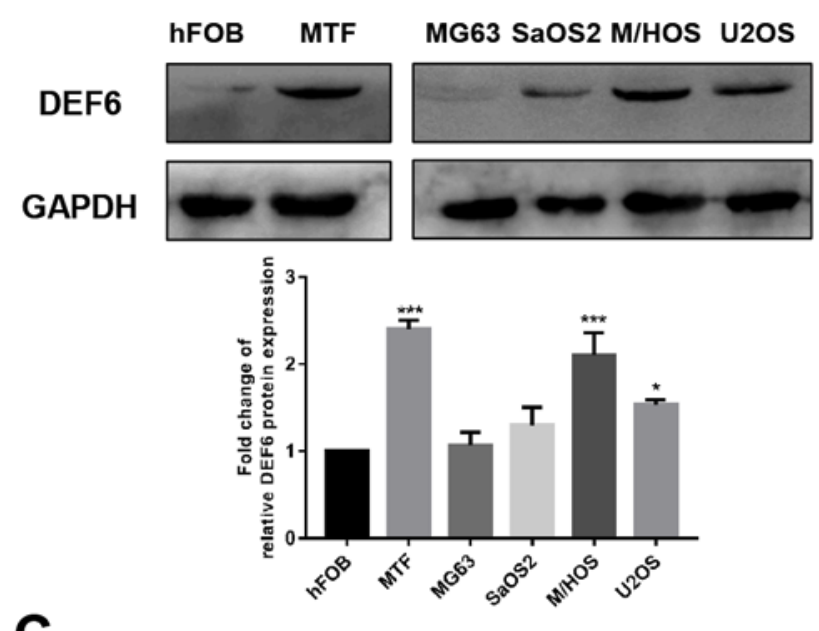

C

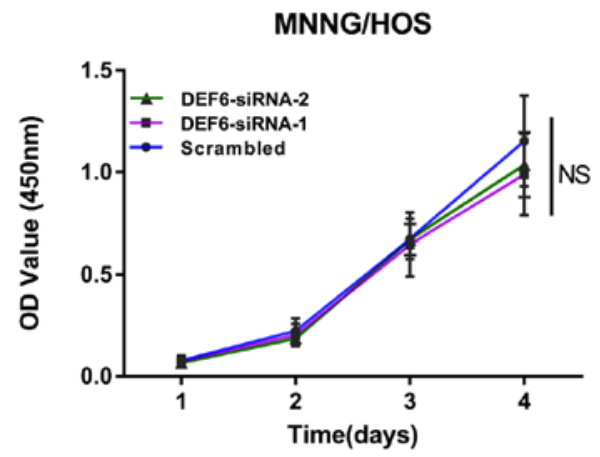

B

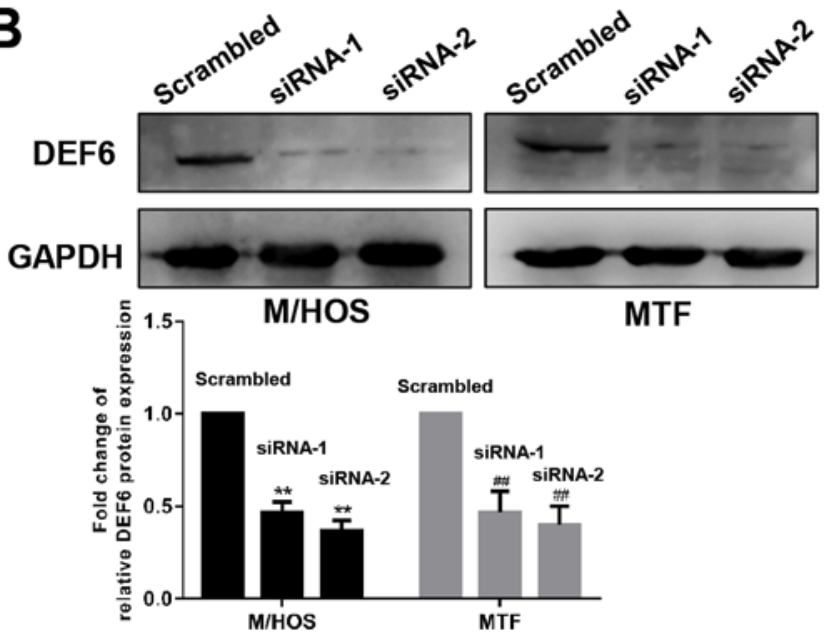

D

\section{Scrambled DEF6-siRNA-1 DEF6-siRNA-2}
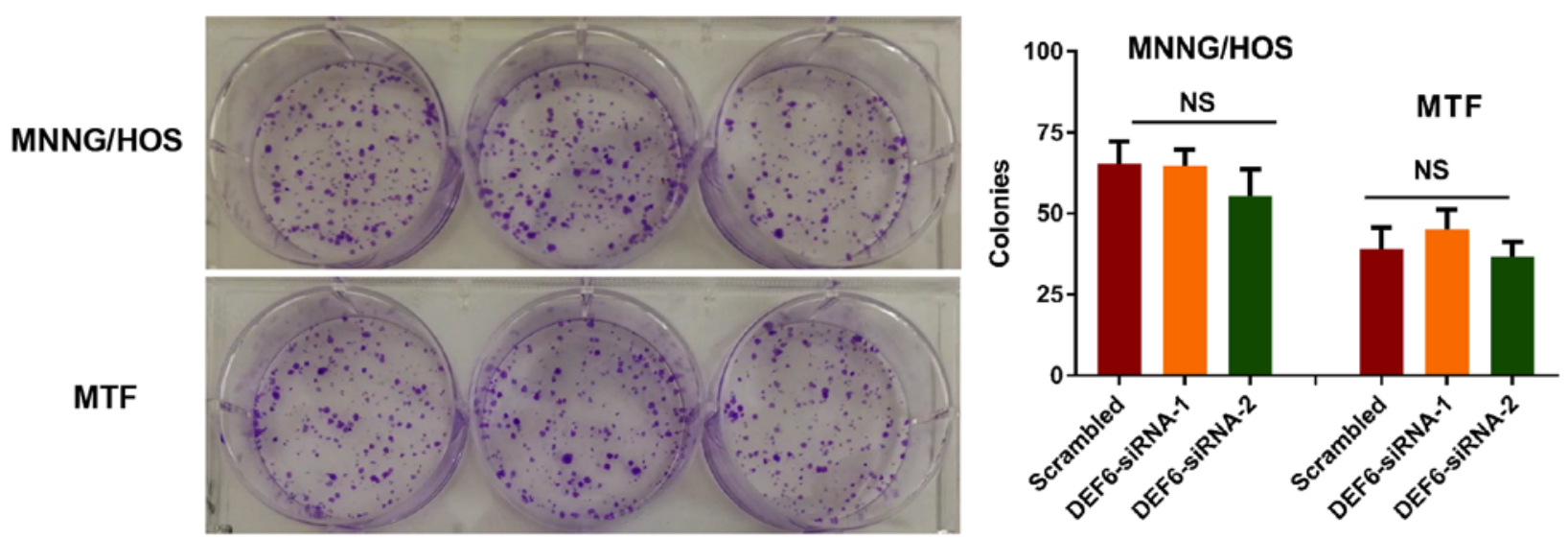

Figure 3. DEF6 is highly expressed in OS cells; however, high DEF6 expression may not contribute to high proliferative ability. (A) DEF6 expression levels in OS and normal osteoblast cell lines. DEF6 was highly expressed in OS cell lines, particularly in MTF and MNNG/HOS cells compared with hFOB1.19 cells. (B) DEF6 expression in MTF and MNNG/HOS cells was successfully knocked down by siRNAs. (C) DEF6 expression knockdown by siRNAs in MTF and MNNG/HOS cells did not significantly affect cell proliferation. (D) DEF6 knockdown in MTF and MNNG/HOS cells did not significantly affect the ability of cell colony formation. ${ }^{*} \mathrm{P}<0.05,{ }^{* * * *} \mathrm{P}<0.001$ vs. the $\mathrm{hFOB}$ group, ${ }^{* *} \mathrm{P}<0.01$ vs. the $\mathrm{M} / \mathrm{HOS}$ scrambled group, ${ }^{\# \#} \mathrm{P}<0.01$ vs. the MTF scrambled group. DEF6, differentially expressed in FDCP 6 homolog; OS, osteosarcoma; MTF, malignant transformed hFOB1.19 cell line; siRNA, short interfering RNA; NS, no significant difference.

knockdown of DEF6 expression had an obvious direct effect on restricting the motility of OS cells in vitro.

DEF6 expression is positively correlated with MMP9 expression in human OS tissues. A total of 58 human OS tissue samples were analyzed to further substantiate the association between MMP9 and DEF6 expression in vivo. Fig. 6 demonstrated the results of positive or negative IHC staining of DEF6 and corresponding
MMP9 levels. A higher positive staining rate $(61.76 \%)$ was detected for MMP9 in patients with OS exhibiting high DEF6 levels compared with patients with OS exhibiting low DEF6 levels (25.00\%; P<0.05). Notably, MMP9-positive OS cells were demonstrated to populate the perivascular space instead of throughout the tissue in OS cases with high DEF6 expression, which may indicate a pre-invasive condition of OS cells (Fig. 6). Further analysis demonstrated that DEF6 expression was 
A
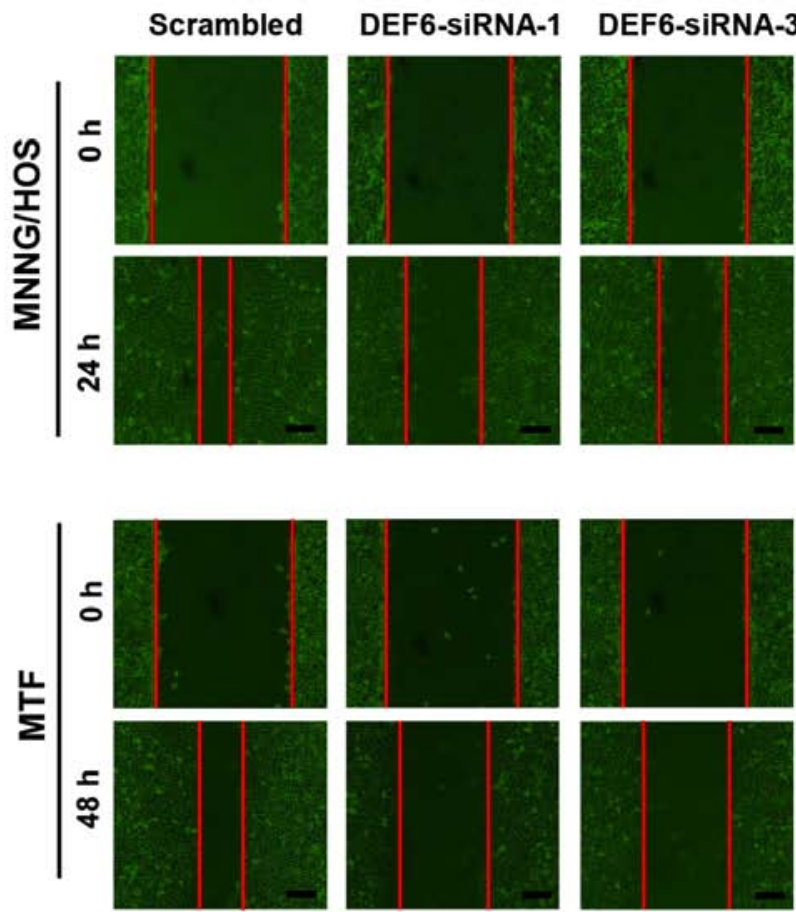
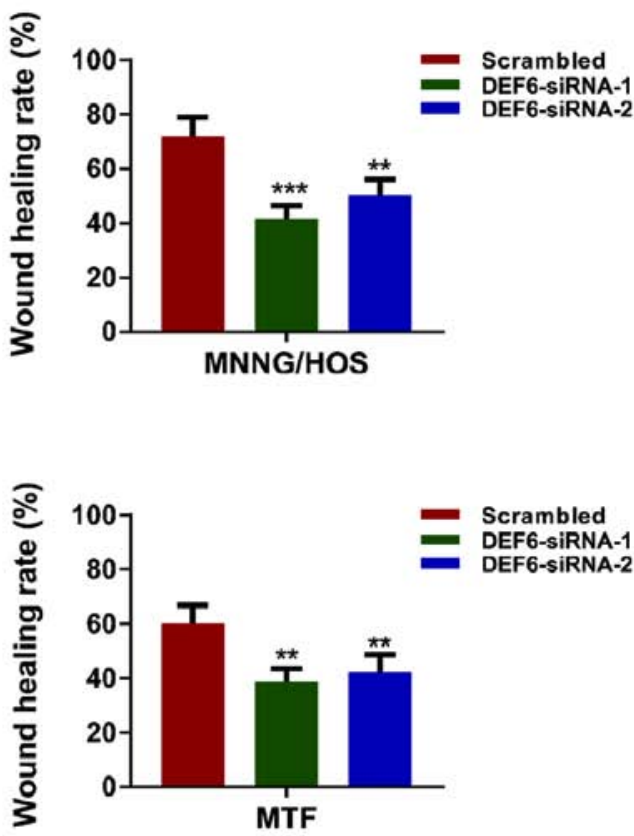
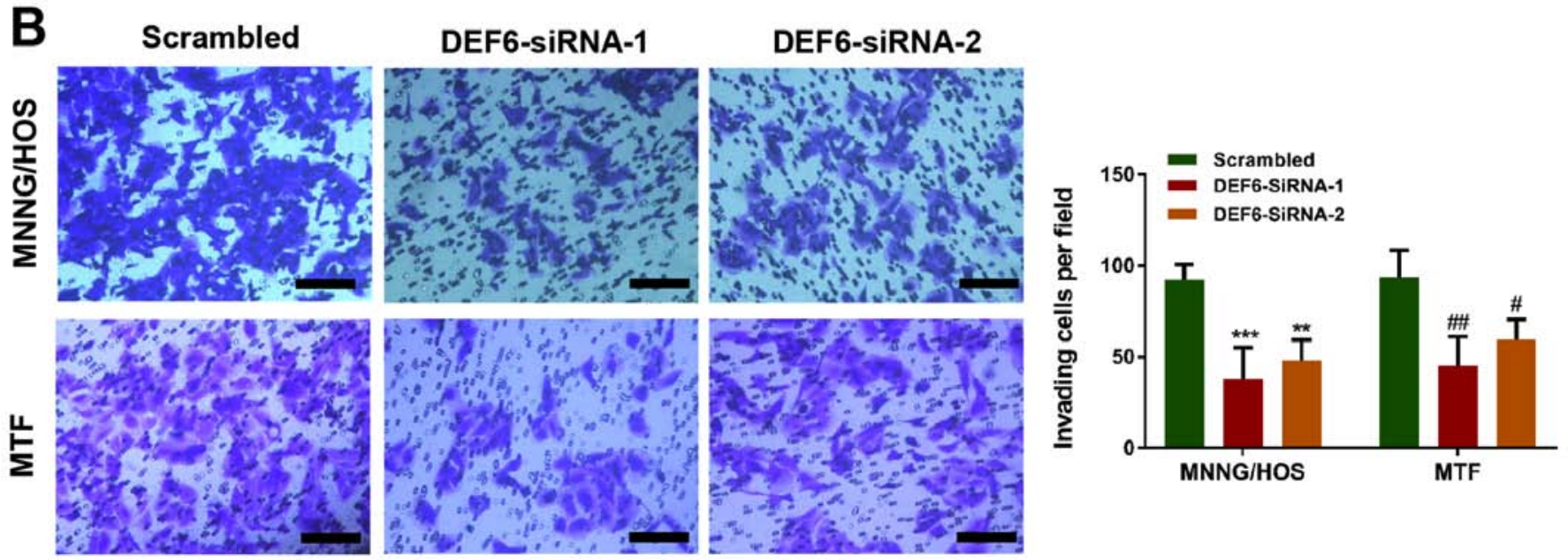

Figure 4. DEF6 knockdown significantly impedes the migration and invasion of osteosarcoma cells. (A) Wound healing assays conducted using MTF or MMNG/HOS cells transfected with scrambled or DEF6 siRNAs. (B) Transwell invasion assay results demonstrated that decreased DEF6 expression decreased the invasion of MTF and MNNG/HOS cells. ${ }^{* *} \mathrm{P}<0.01$ and ${ }^{* * * *} \mathrm{P}<0.001$ vs. the MNNG/HOS scrambled group. ${ }^{*} \mathrm{P}<0.05$ and ${ }^{\# \#} \mathrm{P}<0.01$ vs. the MTF scrambled group. Scale bar, $200 \mu \mathrm{m}$. DEF6, differentially expressed in FDCP 6 homolog; MTF, malignant transformed hFOB1.19 cell line; siRNA, short interfering RNA.

positively correlated with MMP9 expression (Table II). The IHC and in vitro results of the current study indicated that MMP9 serves a crucial role in DEF6-promoted metastasis in OS.

\section{Discussion}

DEF6, also known as IBP, is a pivotal driver responsible for regulating immune cell biology and autoimmunity, and has been frequently been reported to be amplified in several types of cancer $(8,11-23)$. The results of the current study determined whether DEF6 is a novel oncogene in OS. Analysis of 58 patients with OS revealed that DEF6 expression was associated with metastasis and worse clinical stage in OS. Furthermore, high DEF6 levels were positively associated with poor prognosis. DEF6 knockdown demonstrated that metastasis was significantly suppressed in vitro in MTF and MMNG/HOS cells; however, knockdown did not demonstrate a significant difference in cell proliferation. Furthermore, DEF6 expression was positively correlated with MMP9 expression in OS cells and human OS tissues. These data provided preliminary evidence that DEF6 is a potential anti-metastatic target for OS.

Due to the clinical importance of metastasis, a major cause of therapeutic failure in patients with OS (7), early prediction and inhibition of OS metastasis is an appealing curative approach. However, a robust biomarker for predicting metastasis and prognosis in patients with OS does not exist (37). Accumulating evidence suggests that in various types of 


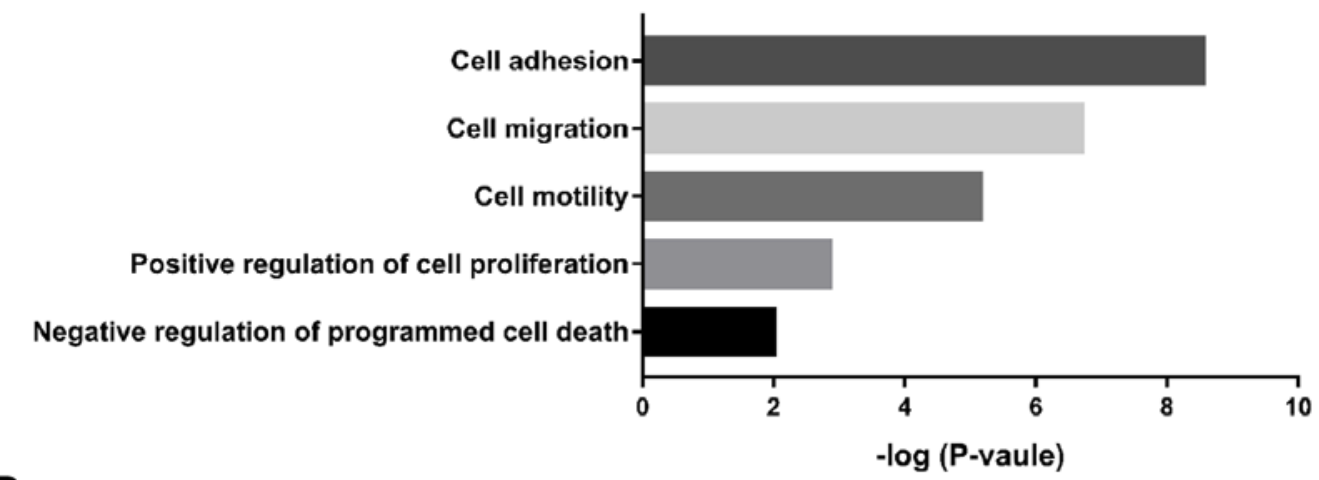

B
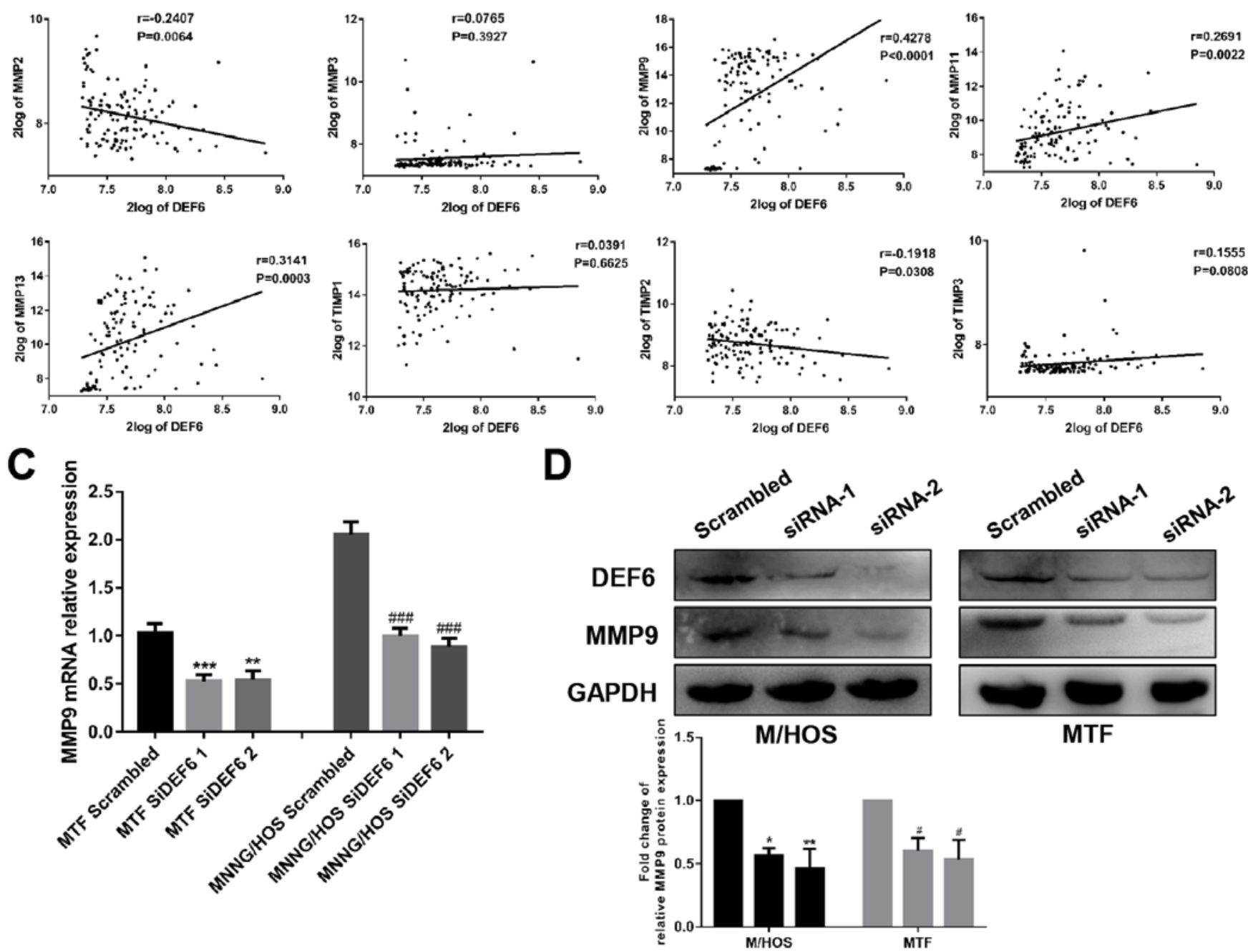

MTF

Figure 5. DEF6 expression is positively correlated with MMP9 expression in human OS cells. (A) Gene ontology categories affected by DEF6 expression in OS tissues. (B) Pearson's correlation analysis between DEF6 and MMPs or TIMPs. (C) mRNA levels of DEF6 and MMP9 in MTF and MNNG/HOS cells, as detected by reverse transcription quantitative PCR. ${ }^{* *} \mathrm{P}<0.01$ and ${ }^{* * *} \mathrm{P}<0.001$ vs. the MTF scrambled group. ${ }^{\# \# \#} \mathrm{P}<0.001$ vs. the MNNG/HOS scrambled group. (D) Western blotting analysis of DEF6 and MMP9 using MTF and MNNG/HOS cells. Cells were treated with scrambled or DEF6 siRNAs. "P<0.05 and ${ }^{* *} \mathrm{P}<0.01$ vs. the M/HOS scrambled group. ${ }^{*} \mathrm{P}<0.05$ vs. the MTF scrambled group. DEF6, differentially expressed in FDCP 6 homolog; MMP9, matrix metallopeptidase P9; OS, osteosarcoma; TIMP, tissue inhibitor of metalloproteinase; MTF, MTF, malignant transformed hFOB1.19 cell line; siRNA, short interfering RNA.

cancer, such as ovarian carcinoma and colorectal cancer, DEF6 is associated with disease progression and poor prognosis $(12,17-20)$. In the present study, the results demonstrated that DEF6 was widely expressed in human OS tissues and that its accumulation was positively correlated with advanced Enneking stage and distant metastases. These results were consistent with those of previous studies $(20,22)$. Moreover, DEF6 was validated to be a potential negative prognostic 

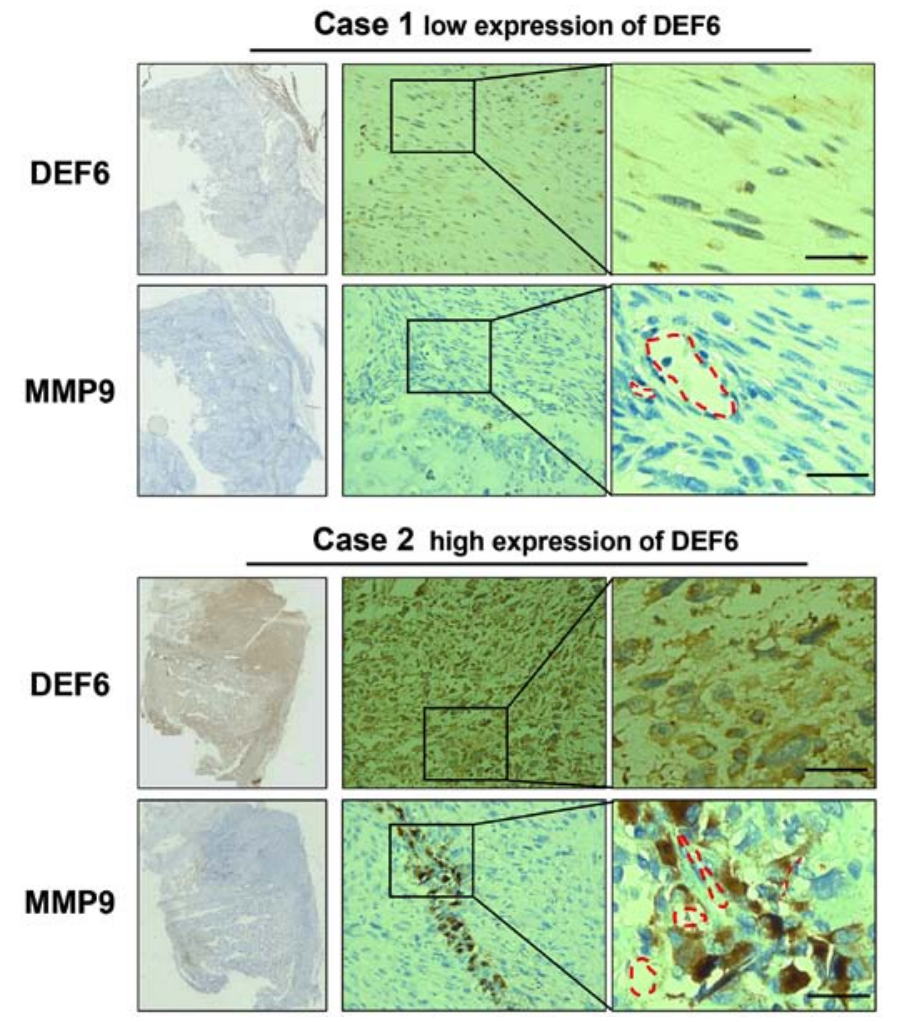

Figure 6. Immunohistochemistry was used to determine DEF6 and MMP9 expression in 58 human osteosarcoma specimens. Two cases with low and high DEF6 expression along with corresponding MMP9 expression. Scale bar, $25 \mu \mathrm{m}$. Red dashed lines indicate the vascular lumen around tumor cells. DEF6, differentially expressed in FDCP 6 homolog; MMP9, matrix metallopeptidase P9.

marker in patients with OS and this result was similar to those of previous studies in ovarian cancer and colorectal cancer $(18,20)$. To the best of our knowledge, the current study is the first to report that increased DEF6 expression may be an indicator of tumor progression and poor prognosis in patients with OS.

DEF6 has been previously reported to serve a role in cancer cell proliferation $(19,22)$. However, the results of current study did not report a significant difference in OS cell proliferation following the knockdown of DEF6 expression via siRNA transfection. Bioinformatics analyses further supported the observation that DEF6 has a negligible impact on OS cell death or growth. Differences in observations between the current and previous studies may be attributed to heterogeneity in different types of cancers.

DEF6 is reportedly an upstream activator of Rho-family GTPases $(14,38)$. In breast cancer cells, DEF6 activates Ras-related C3 botulinum toxin substrate 1 (Rac1), Ras homolog family member A and cell division control protein 42 homolog to promote filipodium and lamellipodia formation, cell migration and MMP secretion (12). Moreover, DEF6 selectively binds to Rac1 to regulate cell morphology (14). These data indicate that DEF6 serves a key role in cancer metastases $(22,39)$. Consistent with these results, the current study demonstrated that DEF6 significantly promoted motility and MMP9 expression in OS cells. Furthermore, a positive correlation was observed between DEF6 and MMP9 expression in human OS tissues.
Table II. Correlation between DEF6 and MMP9 expression in human osteosarcoma.

\begin{tabular}{lcc}
\hline & \multicolumn{2}{c}{ MMP9 expression } \\
\cline { 2 - 3 } DEF6 expression & Positive & Negative \\
\hline High & 21 & 13 \\
Low & 6 & 18 \\
P-value & $0.006^{\mathrm{a}}$ & \\
\hline
\end{tabular}

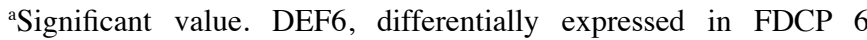
homolog; MMP9, matrix metallopeptidase 9.

The results also demonstrated that tumor tissues with a high expression of DEF6, which may indicate a high risk of OS metastasis, did not contain MMP9-positive OS cells throughout. Instead, MMP9 cells populated the perivascular space. Tumor angiogenesis, one of the most common routes for OS dissemination, has also been demonstrated to promote OS metastasis within the cancer microenvironment $(25,40)$. Thus, the presence of MMP9-positive OS cells around tumor vessels may represent invasion and dissemination through the vessels, which may eventually develop into distant metastasis.

As Rho-family GTPases also reportedly participates in regulating cell migration, cell invasion and actin stress fiber formation in OS cells $(3,9)$. There is a strong possibility that their activation is the potential underlying mechanism through which DEF6 controls OS metastasis. Furthermore, in addition to being a regulator of Rho-family GTPases, DEF6 is reportedly involved in mTOR and Wnt signaling pathway regulation, and DEF6 expression has been directly associated with p53 activation (22,41-43). However, in the current study, whether DEF6 significantly promotes motility in OS cells in vivo and the mechanism via which DEF6 serves a role in OS cell migration and invasion could not be determined; thus, further studies are warranted.

In summary, the current study revealed a new function of DEF6 as an oncogene in OS progression. Upregulated expression of DEF6 may contribute to distant metastasis and poor prognosis in patients with OS, making DEF6 a potential oncogene of OS. Furthermore, the results demonstrated that MMP9 is a potential downstream target of DEF6 and facilitates OS cell invasion. Furthermore, MMP9 expression was positively correlated with DEF6 in OS tissues. Further studies should aim to explore the mechanism by which DEF6 regulates OS metastasis.

\section{Acknowledgements}

The authors of the current study would like to thank Professor Chuanmin $\mathrm{Hu}$ and his research group from the Army Medical University for supplying mouse anti-human DEF6 antibodies. The authors would also like to thank Professor Qing Yin from the Xinqiao Hospital of Army Medical University (Chongqing, China) for her technical and theoretical support, and Mr Jia-Shen Ye and Ms 
Ya-Li Wang from the Xinqiao Hospital of Army Medical University (Chongqing, China) for their assistance with immunohistochemistry techniques.

\section{Funding}

The current study was supported by the National Natural Science Foundation of China (grant nos. 81972519 and 81672653).

\section{Availability of data and materials}

The datasets used and/or analyzed during the current study are available from the corresponding author on reasonable request.

\section{Authors' contributions}

QZ and QG designed the experiments. QZ and GZ conducted the experiments, collected and analyzed the data, and drafted the initial manuscript. YC performed cell culture and some experiments. XT and LL contributed to data collection of human specimens. GZ and QT performed IHC staining analyses. QG provided financial support, administrative support and final approval of the manuscript. All authors read and approved the final manuscript.

\section{Ethics approval and consent to participate}

All human tissues experiments were approved by the Institutional Ethics Committee of Xinqiao Hospital and Army Medical University, Chongqing, China (approval nos. 27-2011 and 2018-069-01). Written informed consent for the experimental studies was obtained from the patients or their guardians.

\section{Patient consent for publication}

Not applicable.

\section{Competing interests}

The authors declare that they have no competing interests.

\section{References}

1. Luetke A, Meyers PA, Lewis I and Juergens H: Osteosarcoma treatment - where do we stand? A state of the art review. Cancer Treat Rev 40: 523-532, 2014.

2. Landis SH, Murray T, Bolden S and Wingo PA: Cancer statistics, 1998. CA Cancer J Clin 48: 6-29, 1998

3. Wang J, Zhang L, Qu R and Huang W: Rho A regulates epidermal growth factor-induced human osteosarcoma MG63 cell migration. Int J Mol Sci 19: 1437, 2018.

4. Siegel RL, Miller KD and Jemal A: Cancer statistics, 2019. CA Cancer J Clin 69: 7-34, 2019.

5. Zhao GS, Zhang Q, Cao Y, Wang Y, Lv YF, Zhang ZS, Zhang Y, Tan QL, Chang Y, Quan XZ, et al: High expression of ID1 facilitates metastasis in human osteosarcoma by regulating the sensitivity of anoikis via PI3K/AKT depended suppression of the intrinsic apoptotic signaling pathway. Am J Transl Res 11: 2117-2139, 2019.

6. Briccoli A, Rocca M, Salone M, Guzzardella GA, Balladelli A and Bacci G: High grade osteosarcoma of the extremities metastatic to the lung: Long-term results in 323 patients treated combining surgery and chemotherapy, 1985-2005. Surg Oncol 19: 193-199, 2010.
7. Mirabello L, Troisi RJ and Savage SA: Osteosarcoma incidence and survival rates from 1973 to 2004: Data from the surveillance, epidemiology, and end results Program. Cancer 115: 1531-1543, 2009.

8. Zhao GS, Gao ZR, Zhang Q, Tang XF, Lv YF, Zhang ZS, Zhang Y, Tan QL, Peng DB, Jiang DM and Guo QN: TSSC3 promotes autophagy via inactivating the Src-mediated PI3K/Akt/mTOR pathway to suppress tumorigenesis and metastasis in osteosarcoma, and predicts a favorable prognosis. J Exp Clin Cancer Res 37: 188, 2018.

9. Liu JL, Li J, Xu JJ, Xiao F, Cui PL, Qiao ZG, Chen XD, Tao WD and Zhang XL: MiR-144 Inhibits Tumor Growth and Metastasis in Osteosarcoma via Dual-suppressing RhoA/ROCK1 Signaling Pathway. Mol Pharmacol 95: 451-461, 2019.

10. Lin H, Hao Y, Zhao Z and Tong Y: Sirtuin 6 contributes to migration and invasion of osteosarcoma cells via the ERK1/2/MMP9 pathway. FEBS Open Bio 7: 1291-1301, 2017.

11. Hotfilder M, Baxendale S, Cross MA and Sablitzky F: Def-2, $-3,-6$ and -8 , novel mouse genes differentially expressed in the haemopoietic system. Br J Haematol 106: 335-344, 1999.

12. Zhang Z, Yang M, Chen R, Su W, Li P, Chen S, Chen Z, Chen A, $\mathrm{Li} \mathrm{S}$ and Hu C: IBP regulates epithelial-to-mesenchymal transition and the motility of breast cancer cells via Rac1, RhoA and Cdc42 signaling pathways. Oncogene 33: 3374-3382, 2014.

13. Manni M, Ricker E and Pernis AB: Regulation of systemic autoimmunity and CD11c(+) Tbet(+) B cells by SWEF proteins. Cell Immunol 321: 46-51, 2017.

14. Oka T, Ihara S and Fukui Y: Cooperation of DEF6 with activated Rac in regulating cell morphology. J Biol Chem 282: 2011-2018, 2007.

15. Joshi RN, Binai NA, Marabita F, Sui Z, Altman A, Heck AJ, Tegnér J and Schmidt A: Phosphoproteomics reveals regulatory T cell-mediated DEF6 dephosphorylation that affects cytokine expression in human conventional T cells. Front Immunol 8: $1163,2017$.

16. Binder N, Miller C, Yoshida M, Inoue K, Nakano S, Hu X, Ivashkiv LB, Schett G, Pernis A, Goldring SR, et al: Def6 restrains osteoclastogenesis and inflammatory bone resorption. J Immunol 198: 3436-3447, 2017

17. Subramanian S, West RB, Marinelli RJ, Nielsen TO, Rubin BP Goldblum JR, Patel RM, Zhu S, Montgomery K, Ng TL, et al: The gene expression profile of extraskeletal myxoid chondrosarcoma. J Pathol 206: 433-444, 2005.

18. Liew PL, Fang CY, Lee YC, Chen CL and Chu JS: DEF6 expression in ovarian carcinoma correlates with poor patient survival. Diagn Pathol 11: 68, 2016.

19. Jian CX, Yang MZ, Li P, Xiong J, Zhang ZJ, Li CJ, Chen A, $\mathrm{Hu}$ CM, Zhou JX and Li SH: Ectopically expressed IBP promotes cell proliferation in oral squamous cell carcinoma. Cancer Invest 30: 748-756, 2012.

20. Zhang Z, Wang Q, Li P, Zhou Y, Li S, Yi W, Chen A, Kong P and $\mathrm{Hu} \mathrm{C}$ : Overexpression of the Interferon regulatory factor 4-binding protein in human colorectal cancer and its clinical significance. Cancer Epidemiol 33: 130-136, 2009.

21. Li P, Zhang Z, Wang Q, Li S, Zhang Y, Bian X, Chen A and $\mathrm{Hu}$ C: The ectopic expression of IFN regulatory factor 4-binding protein is correlated with the malignant behavior of human breast cancer cells. Int Immunopharmacol 9: 1002-1009, 2009.

22. Chen S, Han Q, Wang X, Yang M, Zhang Z, Li P, Chen A, Hu C and Li S: IBP-mediated suppression of autophagy promotes growth and metastasis of breast cancer cells via activating mTORC2/Akt/FOXO3a signaling pathway. Cell Death Dis 4: e842, 2013.

23. Enneking WF, Spanier SS and Goodman MA: A system for the surgical staging of musculoskeletal sarcoma. 1980. Clin Orthop Relat Res: 4-18, 2003.

24. Li Y, Meng G and Guo QN: Changes in genomic imprinting and gene expression associated with transformation in a model of human osteosarcoma. Exp Mol Pathol 84: 234-239, 2008.

25. Gao Z, Zhao GS, Lv Y, Peng D, Tang X, Song H and Guo QN: Anoikisresistant human osteosarcoma cells display significant angiogenesis by activating the Src kinasemediated MAPK pathway. Oncol Rep 41: 235-245, 2019.

26. Livak KJ and Schmittgen TD: Analysis of relative gene expression data using real-time quantitative PCR and the 2(-Delta Delta C(T)) method. Methods 25: 402-408, 2001.

27. Dai H, Huang Y, Li Y, Meng G, Wang Y and Guo QN: TSSC3 overexpression associates with growth inhibition, apoptosis induction and enhances chemotherapeutic effects in human osteosarcoma. Carcinogenesis 33: 30-40, 2012. 
28. Meng G,Lv Y, Dai H, Zhang X and Guo QN: Epigenetic silencing of methyl-CpG-binding protein 2 gene affects proliferation, invasion, migration, and apoptosis of human osteosarcoma cells. Tumour Biol 35: 11819-11827, 2014.

29. Lv YF, Yan GN, Meng G, Zhang X and Guo QN: Enhancer of zeste homolog 2 silencing inhibits tumor growth and lung metastasis in osteosarcoma. Sci Rep 5: 12999, 2015.

30. Guo J, Liu Q, Li Z, Guo H, Bai C and Wang F: miR-222-3p promotes osteosarcoma cell migration and invasion through targeting TIMP3. Onco Targets Ther 11: 8643-8653, 2018.

31. Kunz P, Sähr H, Lehner B, Fischer C, Seebach E and Fellenberg J: Elevated ratio of MMP2/MMP9 activity is associated with poor response to chemotherapy in osteosarcoma. BMC Cancer 16 223, 2016.

32. Li H, Cui J, Xu B, He S, Yang H and Liu L: Long non-coding RNA XIST serves an oncogenic role in osteosarcoma by sponging miR-137. Exp Ther Med 17: 730-738, 2019.

33. Waresijiang N, Sun J, Abuduaini R, Jiang T, Zhou W and Yuan $\mathrm{H}$ : The downregulation of miR125a5p functions as a tumor suppressor by directly targeting MMP11 in osteosarcoma. Mol Med Rep 13: 4859-4864, 2016.

34. Hirahata M, Osaki M, Kanda Y, Sugimoto Y, Yoshioka Y, Kosaka N, Takeshita F, Fujiwara F, Kawai A, Ito H, et al: PAI-1, a target gene of miR-143, regulates invasion and metastasis by upregulating MMP-13 expression of human osteosarcoma. Cancer Med 5: 892-902, 2016.

35. Fan H, Lu S, Wang S and Zhang S: Identification of critical genes associated with human osteosarcoma metastasis based on integrated gene expression profiling. Mol Med Rep 20: 915-930, 2019.

36. Su Y, Wan D and Song W: Dryofragin inhibits the migration and invasion of human osteosarcoma U2OS cells by suppressing MMP-2/9 and elevating TIMP-1/2 through PI3K/AKT and p38 MAPK signaling pathways. Anticancer Drugs 27: 660-668, 2016.
37. Roberts RD, Lizardo MM, Reed DR, Hingorani P, Glover J, Allen-Rhoades W, Fan T, Khanna C, Sweet-Cordero EA, Cash T, et al: Provocative questions in osteosarcoma basic and translational biology: A report from the children's oncology group. Cancer 125: 3514-3525, 2019.

38. Chen Q, Gupta S and Pernis AB: Regulation of TLR4-mediated signaling by IBP/Def6, a novel activator of Rho GTPases. J Leukoc Biol 85: 539-543, 2009.

39. Xu Y, Hou Y, Liu T and Lou G: Overexpression and clinical significance of IBP in epithelial ovarian carcinoma. Oncol Lett 15: 6604-6610, 2018.

40. Wang SW, Liu SC, Sun HL, Huang TY, Chan CH, Yang CY, Yeh HI, Huang YL, Chou WY, Lin YM and Tang CH: CCL5/CCR5 axis induces vascular endothelial growth factor-mediated tumor angiogenesis in human osteosarcoma microenvironment. Carcinogenesis 36: 104-114, 2015.

41. Xu X, Shuen WH, Chen C, Goudevenou K, Jones P and Sablitzky F: Swap70b is required for convergent and extension cell movement during zebrafish gastrulation linking Wnt11 signalling and RhoA effector function. Dev Biol 386: 191-203, 2014.

42. Goudevenou K, Martin P, Yeh YJ, Jones P and Sablitzky F: Def6 is required for convergent extension movements during zebrafish gastrulation downstream of Wnt5b signaling. PLoS One 6: e26548, 2011.

43. Yang M, Yuan F, Li P, Chen $\mathrm{A}, \mathrm{Li} \mathrm{S}$ and $\mathrm{Hu} \mathrm{C}$ : Interferon regulatory factor 4 binding protein is a novel p53 target gene and suppresses cisplatin-induced apoptosis of breast cancer cells. Mol Cancer 11: 54, 2012. International (CC BY-NC-ND 4.0) License. 